\title{
LOS MESOTHERIINAE (MESOTHERIIDAE, NOTOUNGULATA) DEL MIOCENO SUPERIOR DE LA PAMPA, ARGENTINA
}

\author{
Esperanza CERDEÑO' y Claudia I. MONTALVO² \\ ' IANIGLA-CRICYT, Avda. Ruiz Lcal s/n, C.Correo 330. 5500 Mendoza, \\ Argentina. \\ 2Facultad de Ciencias Exactas y Naturales. UNLPam. Avda. Uruguay 151. 6300 \\ Santa Rosa, La Pampa, Argentina.
}

\begin{abstract}
Cerdeño, E. y Montalvo, C.I. 2001. Los Mesotheriinae (Mesotheriidae, Notoungulata) del Mioceno Superior de La Pampa, Argentina. [Mesotheriinae (Mesotheriidae, Notoungulata) from the Late Miocene of La Pampa, Argentina.] Revista Española de Paleontología, 16(1), 63-75. ISSN 0213-6937.
\end{abstract}

\begin{abstract}
More than 180 remains of Mesotheriinae (Mesotheriidae, Notoungulata) from the Upper Miocene of La Pampa Province (Argentina) are described. They come basically from the Cerro Azul Formation and some remains from El Palo Formation, and are identified as Typotheriopsis silveyrai Cabrera, 1937 and Pseudotypotherium subinsigne (Rovereto, 1914). The main character used to differentiate them is the absence or presence of a lingual groove on the P4. The remains come from 18 different localities that have provided a rich mammal assemblage corresponding to the Huayquerian age (Late Miocene). The revision of previous data on mesotherines led us to propose the synonymy of the species Typotheriopsis silveyrai, T. jachalensis and T. vanhouttei, as well as that of Pseudotypotherium carhuense and P. subinsigne, the priority corresponding to $T$. silveyrai and $P$. subinsigne, respectively. The cladistic analysis of the subfamily Mesotheriinae yielded an unique cladogram, which supports all the considered genera as monophyletic taxa. The phylogenetic relationships among the Pseudotypotherium species are not resolved.
\end{abstract}

\section{Keywords: Mesotheriidae, Notoungulata, Upper Miocene, Huayquerian age, La Pampa, Argentina.}

\section{RESUMEN}

Se describen más de 180 restos de Mesotheriidae Mesotheriinae (Notoungulata) del Mioceno Superior de la provincia de La Pampa (Argentina). Proceden básicamente de la Formación Cerro Azul, pero también se ha incluido material de la Formación El Palo, y se identifican como Typotheriopsis silveyrai Cabrera, 1937 y Pseudotypotherium subinsigne (Rovereto, 1914). El carácter principal para diferenciar ambas especies es la ausencia o presencia de surco lingual en el P4. El material procede de 18 localidades diferentes que han proporcionado un rico conjunto de mamíferos atribuible a la edad Huayqueriense (Mioceno Superior). La revisión de los datos existentes sobre los mesoterinos nos lleva a proponer la sinonimia de las especies Typotheriopsis silveyrai, T. jachalensis y $T$. vanhouttei, así como la de Pseudotypotherium carhuense y $P$. subinsigne, correspondiendo la prioridad a $T$. silveyrai y $P$. subinsigne, respectivamente. El análisis cladístico de la subfamilia Mesotheriinae ha proporcionado un único cladograma en el que todos los géneros considerados están justificados como taxones monofiléticos. Las únicas relaciones no resueltas son las existentes entre las especies de Pseudotypotherium.

Palabras clave: Mesotheriidae, Notoungulata, Mioceno Superior, Huayqueriense, La Pampa, Argentina.

\section{INTRODUCCIÓN}

La presencia de mamíferos fósiles en los depósitos continentales del Mioceno Superior de la provincia de La Pampa se conoce desde la década de los 60 (Pascual y Bocchino, 1963; Pascual et al., 1965; Ortega Hinojosa, 1967; Zetti, 1967, 1972; Pascual y Bondesio, 1982). Desde 1986, se han llevado a cabo numerosas campañas de recolección en distintas localidades del centro y este de esta provincia, en rocas asignadas a la Formación Cerro Azul (Fig. 1). La fauna recuperada corresponde a la edadmamífero Huayqueriense (Mioceno Superior) (Fig. 2). Esta fauna se ha correlacionado especialmente con la de la "Formación Epecuén" del oeste de la provincia de Buenos Aires (Pascual y Bocchino, 1963; Pascual et al., 1965; Zetti, 1972) y de Salinas Grandes de Hidalgo (La Pampa) y con la del Huayqueriense de Mendoza y Catamarca (Montalvo et al., 1995, 1996; Goin et al, en prensa). Goin 


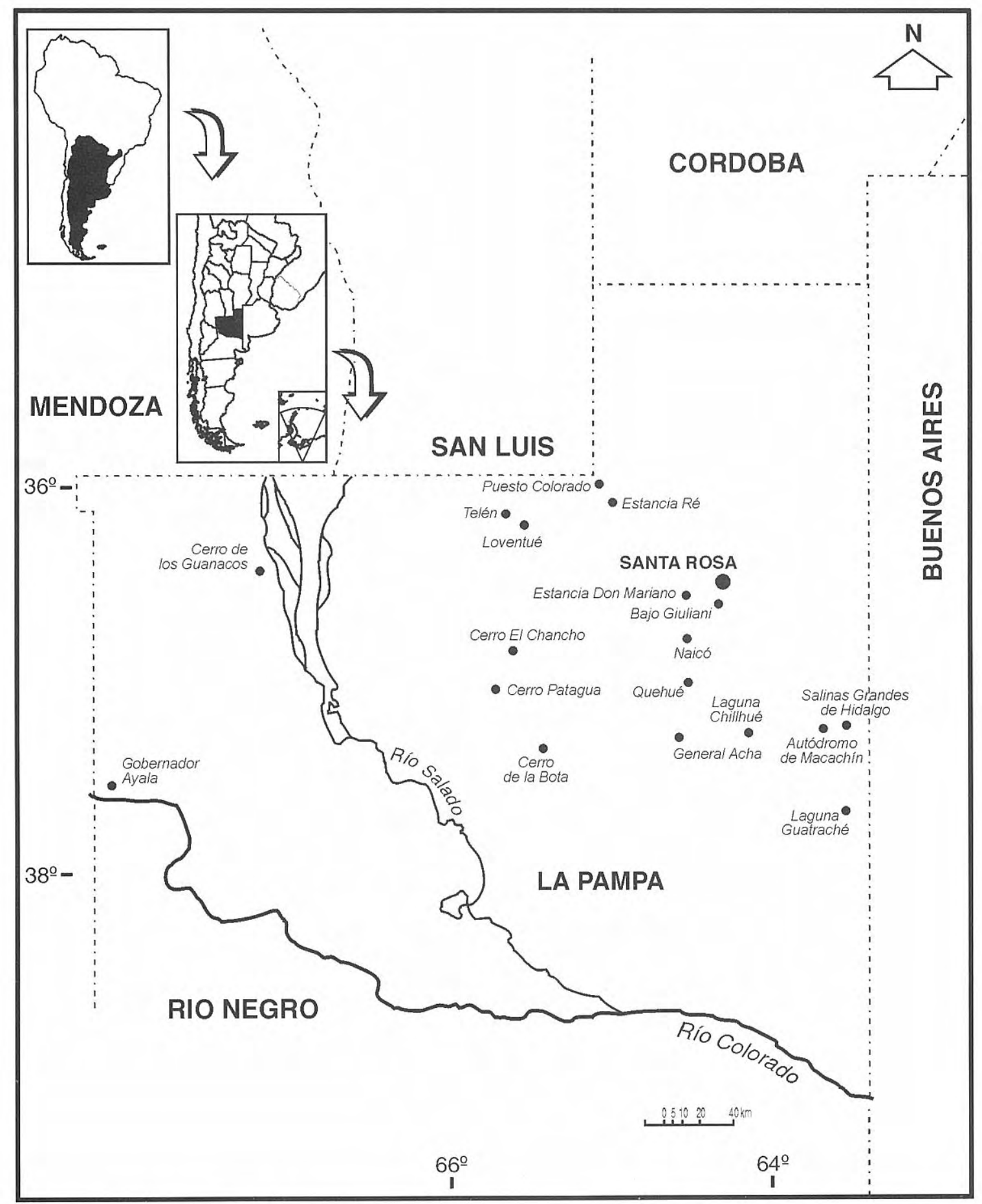

Figura 1. Localización geográfica de los yacimientos huayquerienses de la Provincia de La Pampa. Geographic location of the Huayquerian sites in La Pampa Province.

et al. (en prensa) proponen considerar los niveles fosilíferos de Salinas Grandes de Hidalgo como integrantes de la Formación Cerro Azul, ya que la "Formación Epecuén" nunca fue definida formalmente.

El análisis sedimentológico de la Formación Cerro Azul muestra que la serie comienza con depósitos lagunares, seguidos de potentes estratos de carácter eólico, con muchas evidencias pedogenéticas, sobre los cuales se desarrollaron algunos cursos fluviales esporádicos (Visconti et al., 1996). Los perfiles realizados en varias localidades se integran en el presentado en la Figura 3.

El material estudiado incluye un resto procedente de la Formación El Palo (Uliana, 1979) del oeste de La Pampa, en Gobernador Ayala (departamento Puelén). Los sedimentos fosilíferos corresponden a depósitos fluviales de carácter tabular y gran extensión superficial. Los restos fósiles obtenidos en esta localidad fueron asignados a la edad mamífero Huayqueriense (Franchi et al., 1984). 


\begin{tabular}{|c|c|c|}
\hline $\begin{array}{c}\text { TIEMPO } \\
\text { (Ma) }\end{array}$ & SERIES & $\begin{array}{c}\text { EDADES- } \\
\text { MAMÍFERO }\end{array}$ \\
\hline- & \multirow{2}{*}{ PLIOCENO } & CHAPADMALALENSE \\
\hline 5 & & MONTEHERMOSENSE \\
\hline- & \multirow{6}{*}{$\begin{array}{l}\text { MIOCENO } \\
\text { (MEDIO- } \\
\text { SUPERIOR) }\end{array}$} & HUAYQUERIENSE \\
\hline - & & \\
\hline- & & CHASIQUENSE \\
\hline $\begin{array}{l}- \\
-\end{array}$ & & \\
\hline- & & FRIASENSE \\
\hline $\begin{array}{l}15 \\
- \\
-\end{array}$ & & SANTACRUCENSE \\
\hline
\end{tabular}

Figura 2. Esquema cronoestratigráfico de las edadesmamífero de América del Sur.

Chronostratigraphic scheme of the South American land-mammal ages.

Entre los mamíferos de la Formación Cerro Azul, predominan los notoungulados (Paedotherium minor Cabrera, 1937), acompañados de roedores, xenartros, litopternos, marsupiales y carnívoros, que han sido objeto de diversos estudios parciales (Montalvo y Casadío, 1988; Goin y Montalvo, 1988; Verzi et al., 1991, 1994 1995, 1999; Montalvo et al., 1995, 1996; Esteban y Nasif, 1996; Goin et al., en prensa). En la localidad del valle de Quehué, también se han identificado algunos restos de anfibios y reptiles (Montalvo et al., 1996).

En este trabajo, se describen en detalle los restos de notoungulados de la Familia Mesotheriidae, Subfamilia Mesotheriinae, completando la clasificación previa de los mismos (Montalvo et al., 1995, 1996). Esta subfamilia incluye notoungulados de tamaño mediano a grande, con cráneo de aspecto rodentiforme, de hábitos comparables a los del actual carpincho o capibara (Bond et al., 1995). Los dientes son de crecimiento continuo (Fig. 4) y su fórmula dentaria en los adultos es $1 / 2,0 / 0,2 / 1,3 / 3$ (Pascual et al., 1966). Desde el siglo pasado, se describieron muchas especies de mesotéridos, asignadas a distintos géneros, lo cual dio lugar a una sistemática bastante confusa. Francis (1965) realizó una revisión general del grupo, sinonimizando diversos géneros y aclarando en gran medida el caos existente, aunque sólo en algunos casos lo hizo a nivel de especies. Así, en el Mioceno Superior de Argentina, estarían representados dos géneros (Francis, 1965; Pascual et al., 1966): Typotheriopsis Cabrera y Kraglievich, 1931 y Pseudotypotherium Ameghino, 1904.

En particular para La Pampa, Rusconi (1934) atribuyó un fragmento mandibular procedente de Unanué a Pseudotypotherium insigne, pero este material no ha sido

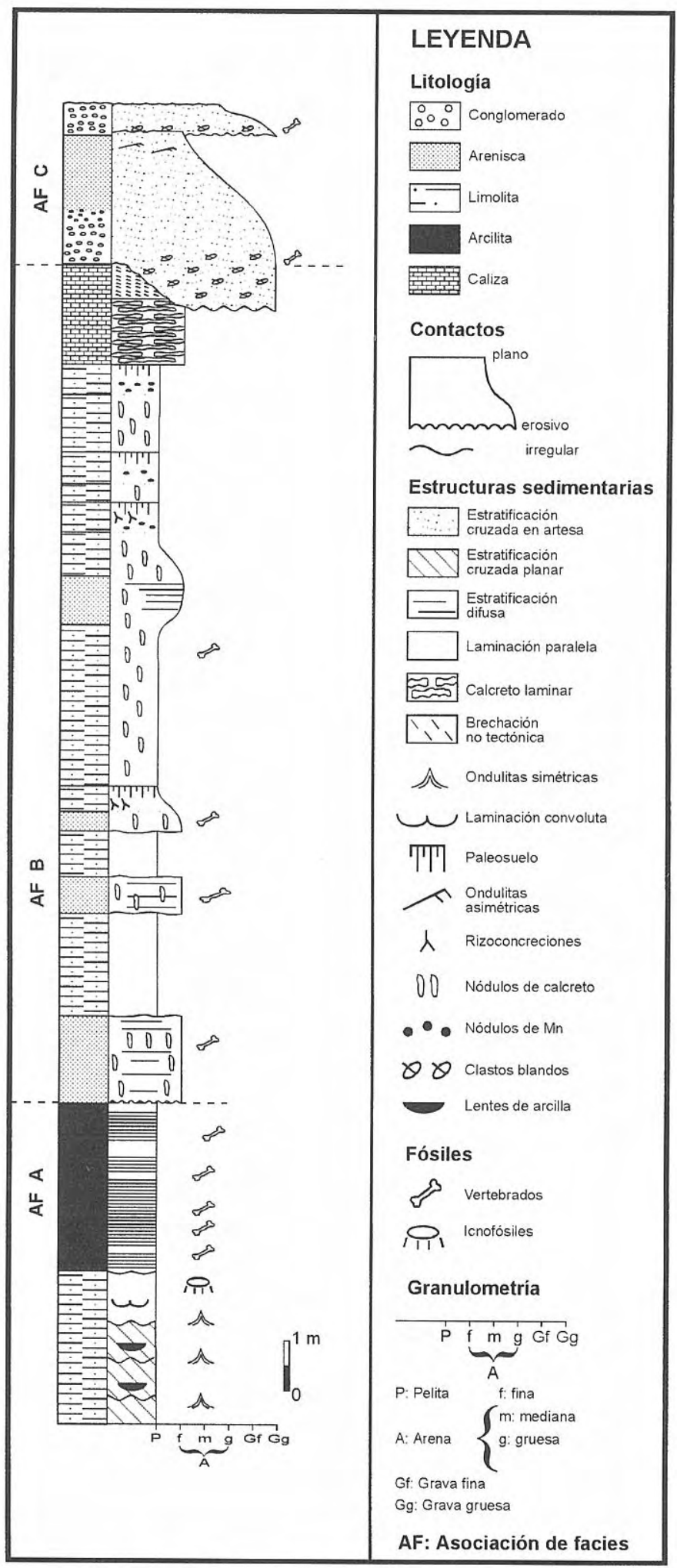

Figura 3. Perfil estratigráfico de la Formación Cerro Azul (modificado de Goin et al., en prensa). Stratigraphic section of the Cerro Azul Formation (modified from Goin et al., in press).

localizado, y fue descrita la especie Typotheriopsis vanhouttei Cattoi, 1943 sobre la base de un material procedente del norte de la provincia. De rocas sedimentarias de la "Formación Epecuén" que afloran en 


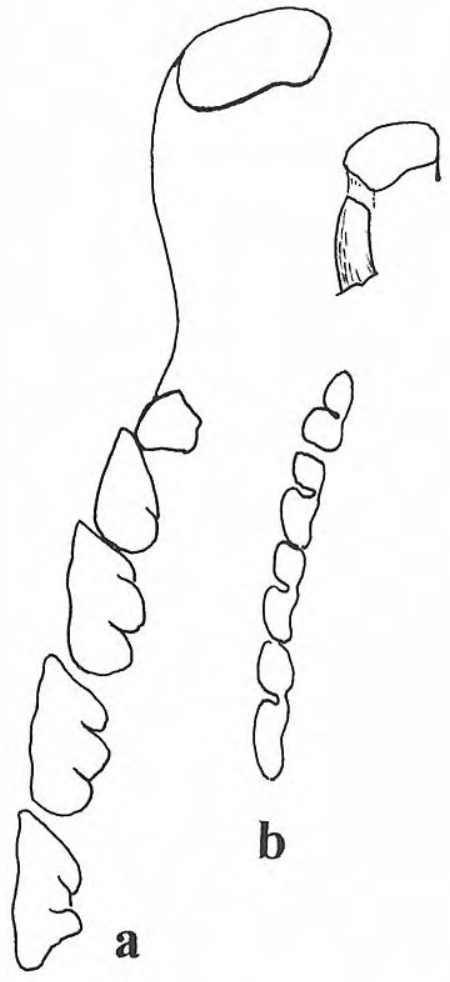

Figura 4. a. Serie dentaria superior de Pseudotypotherium sp., b. Serie dentaria inferior de Pseudotypotherium sp. (modificadas parcialmente de Pascual et al., 1966).

a. Upper tooth series of Pseudotypotherium sp., $b$. Lower tooth series of Pseudotypotherium sp. (partly modified from Pascual et al., 1966).

Adolfo Alsina, al oeste de la provincia de Buenos Aires, proceden varios ejemplares descritos por Cabrera (1939) como Typotheriodon grandis, Typotheriopsis minimus y Pseudotypotherium carhuense y considerados más tarde como una única especie, $P$. carhuense (Francis, 1965). En el Huayqueriense de la provincia de Mendoza, se han descrito Typotheriopsis silveyrai Cabrera, 1937, a partir de un ejemplar procedente de Tupungato, y Pseudotypotherium subinsigne (Rovereto, 1914), con material de las Huayquerías de San Carlos. De niveles coetáneos de la provincia de San Juan, Rusconi (1947) describió la especie Typotheriopsis jachalensis, basado en un fragmento maxilar de Guachi, al norte de Jachal. Para sedimentos huayquerienses de la provincia de Catamarca, se describieron las especies Typotheriopsis internum (Ameghino, 1891) y T. studeri (Moreno y Mercerat, 1891) (ver Marshall y Patterson, 1981).

El material de las formaciones Cerro Azul y El Palo permite reconocer en La Pampa la presencia de los dos géneros Typotheriopsis y Pseudotypotherium.

\section{MATERIAL Y MÉTODOS}

El material estudiado procede de las siguientes localidades (Fig. 1): Bajo Giuliani (departamento Capital), Telén y Loventué (departamento Loventué), Laguna
Chillhué (departamento Guatraché), Autódromo de Macachín y Salinas Grandes de Hidalgo (departamento Atreucó), General Acha, Cerro de la Bota, Cerro Patagua, Quehué y Cerro El Chancho (departamento Utracán), Cerro de los Guanacos (departamento Chical Có), Guatraché (departamento Hucal), Naicó y Don Mariano (departamento Toay), Estancia Ré y Puesto Colorado (departamento Conhelo) y Gobernador Ayala (departamento Puelén).

Se dispone de un conjunto de 186 especímenes, representado fundamentalmente por fragmentos craneales y mandibulares y por dientes aislados, en el que se distinguen dos tallas correspondientes a individuos adultos y a juveniles. Algunos restos no son claramente identificables debido al mal estado de conservación o a su condición fragmentaria. El material estudiado pertenece a la colección de vertebrados fósiles de la Cátedra de Geología Histórica de la Facultad de Ciencias Exactas y Naturales de la Universidad Nacional de La Pampa (GHUNLPam). Se detallan a continuación los restos encontrados en cada localidad, colocando sólo el número de colección y omitiendo la sigla GHUNLPam común a todos:

- Salinas Grandes de Hidalgo: 118/1, P4? iz.; 18/2, i1 iz.?; 118/3, fragmento de molar inferior; 437, I1 der.; 5671, $5931,5932,8303$ y 9180, fragmentos mandibulares iz. (2) y der. (3) con parte de la serie dentaria.

- Autódromo de Macachín: 196, I1 der.; 2430, fragmento mandibular der. con p4-m3.

- Loventué: 152, dos fragmentos mandibulares con d3m3 iz. y d4-m3 der.; 814/1, maxilar der. con P4-M3; 814/2, M2? iz.; 9253, fragmento de maxilar con M3 iz.; 9254, molar superior muy deteriorado; 9280, fragmento de m3 iz.; 9281, fragmento de molar indeterminado; 9282, fragmento de molar superior; 9283, fragmento de m3 iz.; 9284 y 9285 , fragmentos de molares superiores; 9286, fragmento de premaxilar con un resto de incisivo; 9287, fragmento de molar inferior; 9288, fragmento de incisivo; 9290, fragmento mandibular con alvéolo de incisivo, p4 roto y fragmento de molar.

- Telén: 8230, dos fragmentos mandibulares con p4-m3 iz. y dos molares der. rotos; 8232, m3 derecho; 8234, fragmento mandibular con alvéolos de los incisivos, d3 roto y d4; 9048, fragmento de maxilar con M2-M3 iz.; 9068 9072, fragmentos de molares indeterminados; 9096 , fragmento de M3 iz.?; 9111, fragmento lingual de M1?; 9112, fragmento de I1 der.; 9354, 9355 y 9365, fragmentos de molares superiores; 9420, fragmento mandibular con restos de dos molares; 9422, fragmento de p4?; 9457 , fragmento de I1 der.?; 9516, fragmentos de molares; 9522, fragmento mandibular derecho con p4; 12820, fragmento mandibular iz. con $\mathrm{m} 3 ; 12821$, fragmento mandibular der. con m1-m2; 14093 y 14302, fragmentos de molares; 14303, M1 o M2 der.; 14547/1, i1 iz.; 14547/2, M1 o M2?; 14625, fragmento mandibular iz. con m1-m3; 14629, fragmento mandibular iz. con $\mathrm{p} 4$.

- Laguna Chillhué: 157, fragmento de m3 iz.; 158, fragmento de $\mathrm{m} 2$ ? iz.; 160, fragmento mandibular der. con m1-m2 y alvéolo del $\mathrm{m} 3 ; 178$, fragmento de P4? der.; 313, fragmento mandibular der. con m1-m3; 336, M(1 o 2) der.; 337, fragmento de maxilar con P4 y fragmento de M1 der.; 338, fragmento de p4? der.; 364 y 366, fragmentos 
mandibulares der. con restos de $\mathrm{m}(1$ o 2); 376, fragmento de M3 der.; 2033, fragmento de P3 iz.; 2034, fragmento de P3 der.; 2125, fragmento lingual de un M(1 o 2) iz.; 2181, fragmento de molar inferior iz.; 2304, fragmento mandibular con di1, alvéolo del di2, raíz del d3 y d4 fragmentado; 2422, fragmento sinfisario der. con i1 e i2; 2436, fragmento mandibular der. con p4-m1 seccionados; 5436, fragmento lingual de un molar superior; 5571, fragmento de $\mathrm{m} 1$ ? iz.; 5604, fragmento de maxilar con M3 iz.; 5605, fragmento de M1 iz.; 5633, M2 der.; 5998, I1 der.; 5999, fragmento mandibular iz. con molares rotos; M2? y fragmento lingual de M3?; 6960, molar inferior iz.; 6963, fragmento mandibular iz. con dos molares; 8031, fragmento de i1 der.; 8032, P3 iz.; 8033, P3 der.; 8412, fragmento de maxilar con M3 der.; 8416, p4 iz.; 8621, fragmento de I1 ?; 8622 y 8623, fragmentos de molares inferiores iz.; 8624, D4 iz.; 8625, molar superior; 9004, fragmento de molar inferior der.; 9007, fragmento iz. de maxilar con M2-M3 rotos, fragmento mandibular iz. con $\mathrm{m} 3$ y der. con m1-m3; 14442, fragmento de $m 3$ der.; 14443, p4 iz.; 14450, fragmentos de molar.

- General Acha: 327, fragmento de rostro con los incisivos muy fragmentados y P3-P4 der.; 5973/1, M1 der.; 5973/2, fragmento lingual de un molar superior.

- Cerro de la Bota: 347, fragmento de M3 iz.; 348, fragmento mandibular der. con m2-m3; 350, M3 der.; 5304, d4? iz.; 6992, fragmento lingual de molar superior; 6993, fragmento de molar inferior; 8141, fragmento mandibular iz. con fragmento de p4 y m1-m2; 8443, fragmento mandibular der. con m2-m3.

- Quehué: 5925, fragmento mandibular der. con alvéolo del p4 y m1-m3; 8149, M1?; 8628, fragmento mandibular con parte de sínfisis, alvéolo del il y p4-m1; 9152, fragmento mandibular iz. con fragmentos de m1-m3; 9154, maxilar con los dos I1, P3 y M1-M3 der. y P4-M3 iz.; 9677, fragmento mandibular der. con $\mathrm{p} 4-\mathrm{m} 1$ muy rotos $\mathrm{y}$ fragmento mandibular con $\mathrm{m} 2$ ?

- Cerro El Chancho: 8909, I1 der.; 8910, fragmento mandibular iz. con lóbulo posterior de $\mathrm{m} 2$ y m3; 8911 , fragmento mandibular iz. con p4-m 1; 8913, fragmento mandibular con un molar roto.

- Cerro Patagua: 5427, 5429 y 6965, fragmentos mandibulares con restos de molares; 8136, fragmento lingual de M3?

- Bajo Giuliani: 432/1, fragmento de maxilar con M3 der.; 432/2, fragmento de maxilar con M1? iz.; 2184 , fragmento de p4 der.; 2212, fragmento mandibular iz. con $\mathrm{m}(1-2)$; 12856, fragmentos de molar; 14086, fragmento de molar inferior der.

- Cerro de los Guanacos: 813, fragmento de maxilar der. con molares muy fragmentados; 815 , fragmento mandibular der. con $\mathrm{m} 3 ; 816 / 1$, fragmento mandibular con $\mathrm{m} 1-\mathrm{m} 2$ iz.; p4 iz. y fragmento mandibular der. con restos de m1-m2; $816 / 2$, fragmento mandibular der. con i1-i2; un trozo de i2 y el lóbulo posterior del p4, m1 y lóbulo posterior del m2; $816 / 3$, I1 der.; 816/4, fragmento mandibular der. con parte de la raíz del d3 y d4- m1 rotos; 816/5, lóbulo posterior de m3 der.; 9936/1, fragmento lingual de un molar superior der.; 9936/2, fragmento de molar superior; 9936/3, fragmento de M2?; 9936/4, i1 iz.; 9936/5, P3 iz.; 9936/6, I1 iz.; 9936/7, P3 der.; 9936/8, I1 iz.; 9936/9, i2 der.; 9936/10,
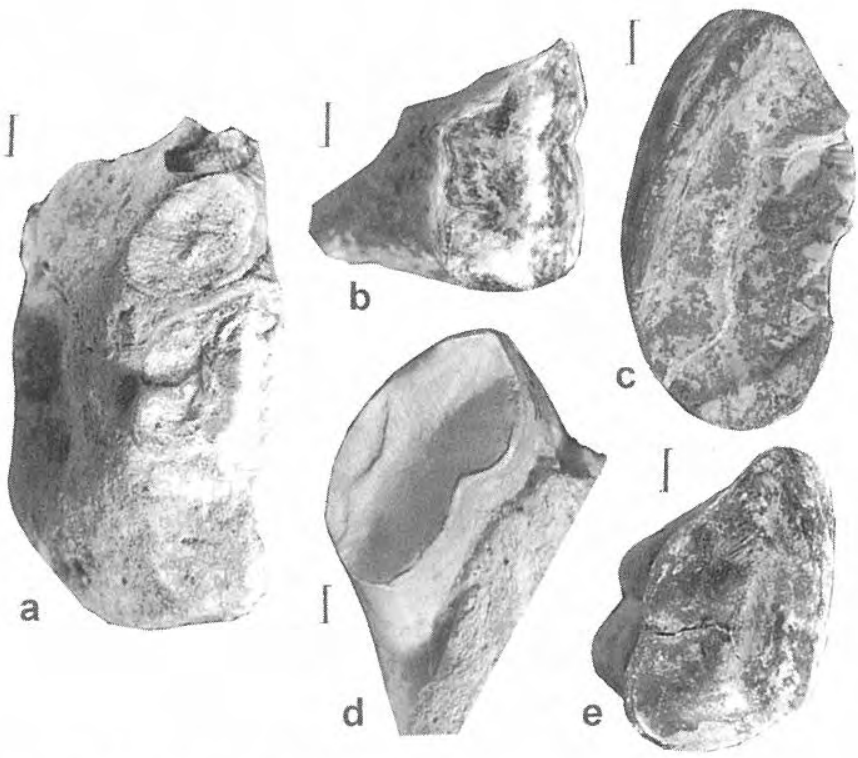

Figura 5. a, D2-D4 izquierdos de Pseudotypotherium, GHUNLPam 21104, Cerro de los Guanacos. b, D4 izquierdo, GHUNLPam 8624, Laguna Chillhué. c, I1 izquierdo, GHUNLPam 816/3, Cerro de los Guanacos. d, I1 derecho, GHUNLPam 196, Autódromo de Macachín. e, P4 izquierdo de Pseudotypotherium, GHUNLPam 9735, Estancia Re. Todos en vista oclusal; b, c y d de Typotheriopsis o Pseudotypotherium. Escalas, $2 \mathrm{~mm}$. $\boldsymbol{a}$, Left D2-D4 of Pseudotypotherium. b, Left D4. $\boldsymbol{c}$, Left I1. d, Right I1. e, Left P4 of Pseudotypotherium. All in occlusal view; $b, c$ and $d$ of Typotheriopsis or Pseudotypotherium. Scales, 2 mm.

fragmento de P3?; 9937, fragmento de maxilar der. con M2 y M3 rotos; 9938, M3 der.; 9939, fragmento mandibular iz. con el lóbulo posterior del m2 y m3; 9941, fragmento mandibular der. con i1-i2 y p4-m1; 9950, fragmento lingual de M(1 o 2) iz.; 9951, fragmento mandibular der. con p4 y m1 muy roto; 9952, fragmento mandibular iz. con m(1 o 2) roto; 9953, m(1 o 2) iz.; 9954, fragmento de maxilar der. con P4-M1; 9955, fragmento mandibular iz. con fragmentos de m2?- m3?; 9956, fragmento de maxilar con M(1 o 2); 9957, P4; 9958, I1 der.; 9959, fragmento de maxilar iz. con M2M3; 9960, molar superior; 9961, fragmento de maxilar der. con P4-M1; 9962, fragmento mandibular iz. con p4-m1; 9963, fragmento mandibular der. con m1?-m2?; 9965 , fragmento de molar superior; 9966, fragmento de maxilar con M2-M3 iz.; 9967, fragmento mandibular iz. con p4-m2; 9968, fragmento mandibular iz. con m1-m3; 9969, fragmento mandibular muy deteriorado; 9970, fragmento mandibular der. con m3 roto; 9971, M(1 o 2) der.; 9972, fragmento mandibular iz. con p4-m1; 19068, fragmento de maxilar con alvéolo de P3 y P4-M1 der.; 19069, fragmento de maxilar con M2-M3 der.; 19070, fragmento de maxilar con M(1 o 2) der.; 19071, fragmento de maxilar con P3; 19072, I1 der.; 19073, fragmento mandibular der. con dos molares rotos; 19074, fragmento mandibular iz. con parte del m3; 21104, fragmento de maxilar iz. con D2-D4. 


\begin{tabular}{|l|c|c|c|c|c|c|c|}
\hline EJEMPLAR & LD2 & AD2 & LP3 & APP3 & LP4 & AAP4 & APP4 \\
\hline MLP 62-XII-4-11* & 6,9 & 6,5 & 9,5 & 8,6 & 13,7 & 8,5 & 7,5 \\
\hline GHUNLPam 8624 * & & & & & 12,7 & 7,25 & 7 \\
\hline N & & & 16 & 16 & 14 & 7 & 14 \\
\hline mínima & & & 10,3 & 10 & 12,6 & 10 & 10,3 \\
\hline media & & & 14,3 & 12,5 & 17,8 & 11,9 & 12,7 \\
\hline máxima & & & 20,3 & 15,25 & 22,8 & 14,3 & 15,7 \\
\hline
\end{tabular}

\begin{tabular}{|c|c|c|c|c|c|c|c|c|c|c|c|c|}
\hline EJEMPLAR & LM1 & AAMI & AMM1 & APM1 & LM2 & AAM2 & AMM2 & APM2 & LM3 & AAM3 & AMM3 & APM3 \\
\hline MLP 62-XII-4-11* & 18,7 & 9,2 & 11,1 & 9 & 18,6 & 9,1 & 11 & 8,3 & 15,6 & 8,2 & 7,6 & 8,2 \\
\hline idem* & 18,6 & 9,3 & 11,2 & 8,9 & & & & & & & & \\
\hline $\mathrm{N}$ & 15 & 13 & 15 & 14 & 16 & 15 & 17 & 17 & 18 & 21 & 19 & 21 \\
\hline mínima & 18,5 & 10,9 & 11,2 & 9 & 18,2 & 9,7 & 10 & 8,5 & 19,9 & 9,6 & 8 & 8,5 \\
\hline media & 23,1 & 12,4 & 12,9 & 11,1 & 23,9 & 12,5 & 12,6 & 10,9 & 24,3 & 12,4 & 11,1 & 12,1 \\
\hline máxima & 28 & 14 & 14,7 & 12,7 & 30,5 & 15 & 15,2 & 13,5 & 30 & 15 & 13,05 & 14,8 \\
\hline
\end{tabular}

Tabla 1. Dimensiones de la dentición superior juvenil (* desglosada) y definitiva (N, número de ejemplares) de los mesotéridos de la Formación Cerro Azul.

Dimensions of the upper juvenile (* separated) and permanent dentition ( $N$, number of specimens) of the mesoterids from the Cerro Azul Formation.

- Laguna Guatraché: 2095, fragmento de m(1 o 2) iz.; 9103, fragmento lingual de molar superior iz.; 19062, fragmento de rama mandibular der., fragmento con los dos incisivos inferiores, otro con p4-m3 der. y otro con $\mathrm{m} 1-\mathrm{m} 2$ iz.

- Naicó: 2110, fragmento de M(1 o 2) der.

- Don Mariano: 12826, cráneo incompleto, mal conservado.

- Estancia Ré: 9715, fragmento de M(1 o 2) iz.; 9734, fragmento de maxilar iz. con M2-M3; 9735, P4 iz.; 9741, fragmento mandibular der. con lóbulo posterior del p4, m1 y fragmento de m2; 9742, I1 iz.; 9743, maxilar con M1-M2 iz. y molares derechos muy deteriorados; 14256/1, P3 iz.; 14256/2, i1 iz.

- Puesto Colorado: 14174, fragmento mandibular iz. con raíz de d3 y d4-m1.

Todos los materiales anteriormente citados provienen de rocas asignadas a la Formación Cerro Azul.

Se describe el material morfométricamente y se compara con diversos restos de mesotéridos del Mioceno Superior, depositados en el Museo de La Plata (MLP) y en el Instituto Miguel Lillo (Tucumán).

Las abreviaturas utilizadas en el texto y las tablas son: A, anchura; AA/AM/AP, anchura anterior/media/posterior; D/d, molar deciduo superior/inferior; der., derecho; I/i, incisivo superior/inferior; iz., izquierdo; L, longitud; M/m, molar superior/inferior; $\mathrm{N}$, número de ejemplares; $\mathrm{P} / \mathrm{p}$, premolar superior/inferior.

Todas las dimensiones se expresan en milímetros.

\section{DESCRIPCIÓN COMPARATIVA}

Dada la homogeneidad general existente en la dentición de los mesotéridos y la falta de rasgos diagnósticos en muchos de los ejemplares estudiados, preferimos hacer una descripción conjunta de los restos, indicando las diferencias apreciables entre ellos y señalando las afinidades con el material de comparación.

\section{Dentición superior decidua}

A la dentición decidua superior se atribuyen los ejemplares GHUNLPam 21104 y 8624. El primero corresponde a un fragmento de maxilar con el D2 roto y D34. El D3 tiene aspecto de premolar y es de sección subtriangular, el D4 es francamente molariforme, con un surco desarrollado que en su fondo presenta una pequeña bifurcación interna (Fig. 5a). El ejemplar GHUNLPam 8624 es un diente aislado, caracterizado por un parastilo largo y saliente, bien delimitado por un surco en el ectolofo (Fig. $5 b$ ); lingualmente tiene un surco fino y corto que lo divide en dos lóbulos; el anterior, a su vez, tiene una suave ondulación lingual; la cara lingual forma ángulos rectos anterior y posteriormente, dando un aspecto general cuadrangular del que sobresale el parastilo anterolabialmente. Parece tratarse de un D4, pero es distinto del de Pseudotypotherium carhuense (MLP 37-III-7-1 y 5) de Adolfo Alsina (Buenos Aires), en el que los lóbulos están bien definidos; coinciden, en cambio, en el gran desarrollo del parastilo y en las dimensiones (Tabla 1). El ejemplar MLP 62-XII-4-11 de Valle Argentino (localidad que corresponde con la aquí denominada Laguna Chillhué) es una serie D2-D4-M1 (Tabla 1), cuyo D4 es similar al anteriormente descrito. El D2 es corto y subtriangular, con el pliegue del paracono formando un ángulo en el ectolofo. El D3 es menos triangular y con la cara lingual más larga y sin surco. Según Francis (1965), los D2 y D3 de Typotheriopsis son subtriangulares y no tienen surco lingual, mientras que en Pseudotypotherium la presencia de este surco es variable; el D4 de Typotheriopsis y Pseudotypotherium es subcuadrangular y trilobulado, aunque los lóbulos pueden estar reducidos.

\section{Dentición superior definitiva (Tablas 1 y 3 )}

Los incisivos superiores (I1) se sitúan muy próximos a la línea media longitudinalmente, no transversalmente como en Mesotherium. Tienen un surco lingual marcado que se ubica en posición media (ej. GHUNLPam 816/3, 9112 y 9457; 


\begin{tabular}{|c|c|c|c|c|c|c|c|c|c|c|c|c|c|c|}
\hline EJEMPLAR & Ld3 & $\mathrm{Ad} 3$ & Lp4 & AAp4 & APp4 & Lm1 & AAml & $\mathrm{APml}$ & Lm2 & AAm2 & $\mathrm{APm} 2$ & Lm3 & AAm3 & $\mathrm{APm} 3$ \\
\hline GHUNLPam $152^{*}$ & 5,8 & 5,2 & 10,4 & 6,45 & 7,8 & 14 & 7,2 & 7,8 & 15 & 6,4 & 6,25 & & & 5,7 \\
\hline GHUNLPam 152* & & & & & & 14 & 6,9 & 7 & & & & & & \\
\hline GHUNLPam 2304 * & 5 & 4,5 & & 5,3 & 6,65 & & & & & & & & & \\
\hline GHUNLPam $5304^{*}$ & & & 9,4 & 4,4 & 5,8 & & & & & & & & & \\
\hline GHUNLPam $8234^{*}$ & 6,8 & & 10,7 & 4,5 & 7 & & & & & & & 29,6 & 7,7 & 8,2 \\
\hline GHUNLPam 348 * & & & & & & 12,5 & 5,5 & 5,3 & 16,8 & 4,7 & 4,8 & & & \\
\hline GHUNLPam $8443^{*}$ & & & & & & 11,5 & 5,8 & 6,2 & 15,2 & 5 & 4,9 & & & \\
\hline GHUNLPam 9522 * & & & 10,4 & 5,8 & 8,3 & & & & & & & & & \\
\hline GHUNLPam 9963 * & & & & & & 15,4 & 6 & 7 & & & & & & \\
\hline GHUNLPam 9968 * & & & & & & & & 8,5 & 15,2 & & 6 & 19,2 & 5 & 4,7 \\
\hline $\mathrm{N}$ & & & 20 & 19 & 21 & 29 & 32 & 33 & 17 & 19 & 19 & 14 & 16 & 16 \\
\hline minima & & & 12 & 6,2 & 8,45 & 13,6 & 5,4 & 8 & 17,9 & 6,4 & 6,8 & 15,16 & 5,3 & 5 \\
\hline media & & & 16,1 & 7,4 & 10,3 & 19,0 & 8,7 & 10,3 & 22,8 & 8,9 & 9,7 & 26,1 & 7,9 & 8,2 \\
\hline máxima & & & 20,4 & 9,4 & 12,5 & 22,5 & 11,3 & 12,4 & 27,5 & 10,6 & 11,1 & 32,1 & 10,2 & 11,3 \\
\hline
\end{tabular}

Tabla 2. Dimensiones de la dentición inferior juvenil (* desglosada) y definitiva (N, número de especímenes) de los mesotéridos de la Formación Cerro Azul.

Dimensions of the lower juvenile (* separated) and permanent dentition ( $N$, number of specimens) of the mesoterids from the Cerro Azul Formation.

Fig. 5c) o ligeramente desplazado hacia la mitad anterior (ej. GHUNLPam 196, 327, 437, 5998, 8621, 8909, 9936/8 9958 y 19072; Fig. 5d).

El P3 es subtriangular, corto, con un fino surco parastilar y otro esbozo de surco en posición media del ectolofo. El P4 es parecido, también con surco parastilar. Ambos se asemejan mucho, aun siendo algo más grandes, a los del ejemplar MLP 36-XI-10-2 de Typotheriopsis silveyrai de Tupungato (Mendoza). Algunos ejemplares (ej., GHUNLPam 178, 2033, 8032, 9735, 9957, 12826) muestran unos premolares (P3 y P4) también subtriangulares, pero mucho más alargados, con la longitud claramente mayor que la anchura y con una talla general mayor que en el caso anterior. En la muestra estudiada, particularmente los P4, presentan dos morfologías diferenciables. La mayoría de los P4 disponibles tienen un esbozo de surco lingual, sin llegar al desarrollo que se aprecia en los de Pseudotypotherium que son claramente bilobulados (Francis, 1965; Pascual et al., 1966). El P4 GHUNLPam 9735 (Fig. 5e) tiene un surco bien marcado, en tanto que en el ejemplar GHUNLPam 814/1 el P4 presenta un surco desarrollado y con una pequeña bifurcación interna, tal como fue descrita por Francis (1965), carácter que se observa también en el ejemplar MLP 121695 de Pseudotypotherium de Monte Hermoso.

Por otro lado, es de destacar que todos los ejemplares de La Pampa revisados en las colecciones del MLP presentan los P4 bilobulados, con un surco lingual bien patente. Todos ellos proceden de Salinas Grandes de Hidalgo, salvo el ejemplar MLP 71-IX-3-1, de Gobernador Ayala (correspondiente a la Formación El Palo). Entre los primeros, el MLP 65-VII-29-33 destaca además por el alargamiento del lóbulo posterior en los dos P4 del ejemplar. En menor medida, también queda un poco destacado dicho lóbulo en el P4 del MLP 65-VII-29-32.

Estos P4 bilobulados ponen en evidencia la presencia de Pseudotypotherium en estos dos yacimientos, a los que ahora se agregan materiales de Cerro de los Guanacos, Laguna Chillhué y Estancia Ré. Por otro lado, los P3 de estos ejemplares son subtriangulares, pero de contorno más convexo que los atribuidos a Typotheriopsis. En las

\begin{tabular}{|l|c|c|}
\hline & \multicolumn{2}{|c|}{ I1 } \\
\hline EJEMPLAR & L & A \\
\hline GHUNLPam 196 & 17,2 & 9 \\
\hline GHUNLPam 327 & 25 & \\
\hline GHUNLPam 437 & 27,2 & 15,2 \\
\hline GHUNLPam 816/3 & 20 & 12,9 \\
\hline GHUNLPam 5998 & 17,2 & 10,5 \\
\hline GHUNLPam 8909 & 23,85 & 12 \\
\hline GHUNLPam 9154 & 25 & 15,05 \\
\hline GHUNLPam 9154 & 25 & 14,2 \\
\hline GHUNLPam 9742 & 29,9 & 15 \\
\hline GHUNLPam 9936/8 & 22 & 15,3 \\
\hline GHUNLPam 9958 & 23,2 & 15,55 \\
\hline GHUNLPam 14547 & 25 & 13,45 \\
\hline
\end{tabular}

\begin{tabular}{|l|c|c|}
\hline & \multicolumn{2}{|c|}{ i1 } \\
\hline EJEMPLAR & L & $\mathrm{A}$ \\
\hline GHUNLPam 816/2 & 17,4 & 11,8 \\
\hline GHUNLPam 2304 & 12,4 & 7,3 \\
\hline GHUNLPam 9936/4 & 17,45 & 12 \\
\hline GHUNLPam 9936/6 & 19 & 13 \\
\hline GHUNLPam 9941 & 17,4 & 11,7 \\
\hline GHUNLPam 14256 & 14,4 & 8,3 \\
\hline GHUNLPam 19062 & 23,7 & 14,4 \\
\hline & & i2 \\
\hline EJEMPLAR & L & $\mathrm{A}$ \\
\hline GHUNLPam 816/2 & 7,3 & 8,75 \\
\hline GHUNLPam 19062 & 9,4 & 8,2 \\
\hline
\end{tabular}

Tabla 3. Dimensiones de los incisivos superiores (I1) e inferiores (i1 e i2) de los mesotéridos de la Formación Cerro Azul.

Dimensions of the upper incisors (II) and lower incisors (i1-i2) of the mesoterids from the Cerro Azul Formation. 

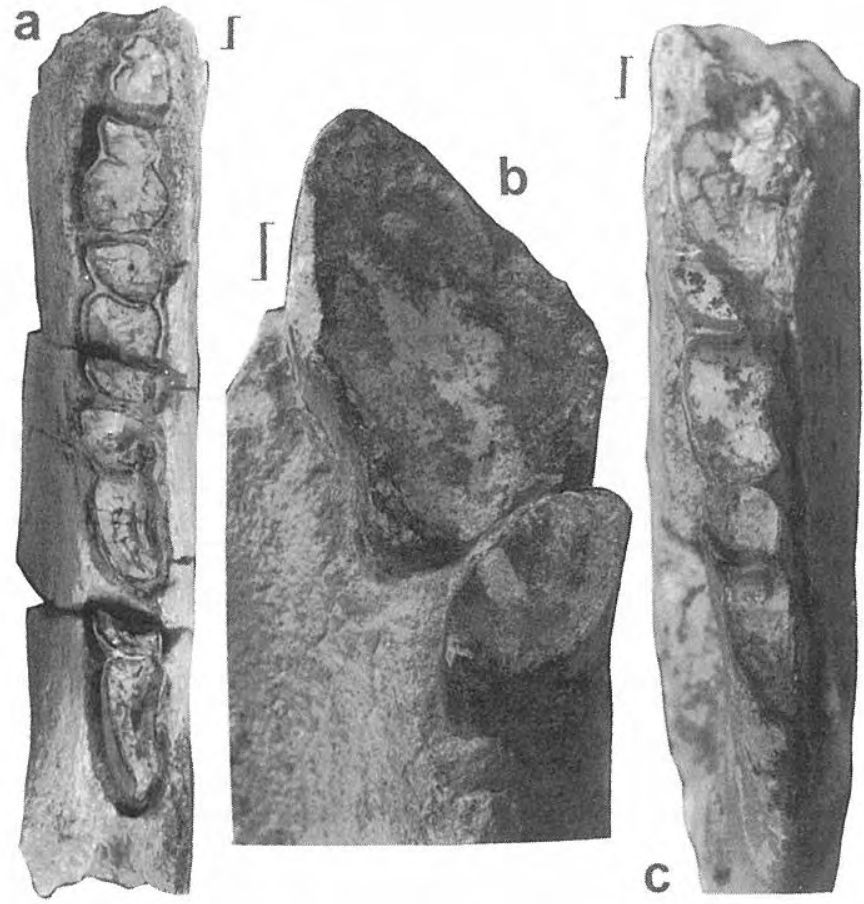

Figura 6. a, d3-m3 izquierdos de Typotheriopsis o Pseudotypotherium, GHUNLPam 152, Loventué, b i1-i2 derechos de ?Pseudotypotherium, GHUNLPam 816/2, Cerro de los Guanacos. c, m1-m3 izquierdos de ?Pseudotypotherium, GHUNLPam 9968, Cerro de los Guanacos. Todos en vista oclusal. Escalas, $2 \mathrm{~mm}$.

$\boldsymbol{a}$, Left d3-m3 of Typotheriopsis or Pseudotypotherium; $\boldsymbol{b}$, Right il-i2 of ?Pseudotypotherium; $\boldsymbol{c}$, Left $m 1-m 3$ of ?Pseudotypotherium. All in occlusal view. Scales, $2 \mathrm{~mm}$.

recolecciones recientes en Salinas Grandes de Hidalgo, no se ha recuperado ningún resto con dentición superior.

Los M1 y M2, trilobulados, son similares entre sí, con el lóbulo anterior estrecho y el lóbulo medio ancho y un poco saliente lingualmente. El M3 tiene el parastilo ancho y saliente, marcado por un surco labial; un surco más suave aparece en el extremo posterior, por delante del metastilo que es ancho y saliente; el lóbulo anterior es largo, estrecho y dirigido hacia atrás; el lóbulo medio está bien desarrollado, pero es algo más corto que los otros dos. Hay dos ejemplares de Salinas Grandes de Hidalgo (MLP 65-VII-29-32 y 33), ya comentados antes por la morfología de sus P4, que tienen los M3 con el lóbulo medio especialmente corto.

Entre todo el material consultado de yacimientos de diversas edades, existen especímenes cuyos M3 muestran el lóbulo medio más "encerrado" entre los otros dos (ej., tipo de Typotherium histatum MLP 43-VII-10-1 de Miramar; $T$. bravardi MLP 12-1689 de Monte Hermoso), pero este rasgo parece responder más a la edad y variabilidad individual que a diferencias taxonómicas.

\section{Dentición inferior decidua}

Existen varios fragmentos mandibulares que conservan los dientes deciduos (Tabla 2). En el GHUNLPam 2304, se conserva, además de restos de d3 y d4, el di 1 cuadrangular, poco apuntado antero-labialmente, con surco lingual amplio y estrías labiales longitudinales.

Tanto en aquel ejemplar como en GHUNLPam 152 (Fig. $6 a)$, el d3 es un diente corto, triangular, ancho posteriormente; el lóbulo anterior forma una arista labial aguda. El d4 es más cuadrangular, bilobulado, pero también con dos aristas labiales agudas en el lóbulo anterior que es mucho más corto que el posterior; el surco labial está bien marcado pero apenas alcanza la mitad de la anchura oclusal en ese punto, al mismo nivel que el pequeño pliegue lingual. El ejemplar GHUNLPam 14174 es un fragmento mandibular con dos dientes rotos y un resto de raíz del diente anterior a ellos; parecen corresponder a la raíz del d3, d4 y m1, pero en este caso el d4 tiene un surco labial más profundo; su talla es similar a la del ejemplar GHUNLPam 2304.

En los nuevos ejemplares, los d3 son similares al de Pseudotypotherium carhuense (MLP 37-III-7-1 y 5), en tanto los d4 son más grandes y tienen el surco un poco menos profundo que el de ese ejemplar. Por otra parte, la descripción de Francis (1965) de los d3 y d4 de Typotheriopsis coincide tanto con la del ejemplár GHUNLPam 152 como con la de estos dientes en Pseudotypotherium, por lo que no es definitiva la adscripción de nuestro material a uno u otro género. Francis (1965) considera como un juvenil de Typotheriopsis (con dudas) el "Acrotypotherium" descrito por Rusconi (1936: fig. 1); según la figura de este autor, el d3 es más cilíndrico y el d4 es más alargado y elíptico que los otros ejemplares estudiados.

\section{Dentición definitiva inferior (Tablas 2 y 3 )}

El il es cuadrangular, trapezoidal, con suave concavidad lingual y el extremo mesio-lingual apuntado. En general, presentan un suave surco en la cara posterior, aunque hay ejemplares (ej. MLP 65-VII-29-31) en los que tanto el surco lingual como el posterior son más patentes y podrían corresponder a Pseudotypotherium, de acuerdo con la descripción de Francis (1965). Los ejemplares de i1 e i2 hallados asociados entre el material estudiado muestran una proporción entre ambos mayor de 2/1 (ej. GHUNLPam $816 / 2$ y 9941 ; Fig. 6b), lo cual también indicaría que se trata de dicho género y no de Typotheriopsis. Sin embargo, comparando con $T$. chasicoensis (holotipo MLP 12-1666 y MLP 67-XII-27-1), la diferencia es mínima en cuanto a la proporción observada. Aparte del material tipo de Typotheriopsis silveyrai (MLP 36-XI-10-2; Cabrera, 1937) otros restos procedentes de Tupungato (Mendoza), como MLP 40-III-11-1 y MLP 37-III-1-2, están muy mal conservados e incompletos, pero en el primero se observa un i1 trapezoidal con surco posterior y bastante aplanado lingualmente y la proporción i1/i2 es también algo mayor de $2 / 1$. Respecto a este carácter, entre los materiales de La Pampa, sólo el ejemplar MLP 62-XII-4-15 de Laguna Chillhué muestra una proporción de $3 / 1$. El mismo valor se encuentra en el MLP 48-XII-16-225 de Monte Hermoso, mientras que el MLP 46-V-13-5 también de este yacimiento alcanza una relación de 5/1, característica de Mesotherium del Pleistoceno. Los tres especímenes mencionados son atribuibles a Pseudotypotherium, lo cual indica que el gran 
ensanchamiento relativo de los il empezó a manifestarse ya en el Mioceno Superior y el Plioceno, generalizándose en el género pleistoceno. Por otro lado, en el MLP 40-III-11-1 procedente de Tupungato (Mendoza), se observa un i1 trapezoidal con surco posterior y bastante aplanado lingualmente y la proporción i1/i2 es algo mayor de $2 / 1$. Esta proporción también se ha observado en dos ejemplares depositados en el Instituto M. Lillo de Tucumán, uno de ellos de Catamarca y otro sin procedencia.

El p4 es subtriangular, con el surco labial corto, más o menos hasta la mitad de la anchura; el lóbulo anterior es apuntado mesialmente; se observa cierta variación en la convexidad de la pared lingual de los lóbulos (más aplanada en GHUNLPam 816/1 y más convexa en GHUNLPam 2430). Estos dos ejemplares difieren además en la talla, mayor en el GHUNLPam 2430. El GHUNLPam 816/1 tiene dimensiones similares a las del holotipo de "Typotheriodon grandis" (= Pseudotypotherium carhuense).

Entre los molares inferiores definitivos, algunos son estrechos y de talla relativamente pequeña, que interpretamos como pertenecientes a individuos aún jóvenes. Entre ellos, los ejemplares GHUNLPam 348 y GHUNLPam 9968 (Fig. 6c) muestran el $\mathrm{m} 2$ con su extremo posterolingual formando una pequeña columnita, que también se observa en el ejemplar juvenil de $P$. carhuense (MLP 37-III7-1), al cual se acerca también por la talla. En general, los molares, tanto de los individuos juveniles como de los adultos, muestran una arista antero-labial aguda; el surco labial es muy profundo, casi hasta la cara lingual; el lóbulo posterior tiene una suave concavidad lingual y es relativamente más ancho y corto en el $\mathrm{m} 1$ que en el $\mathrm{m} 2$ y mucho más estirado en el m3 (más apuntado en los juveniles). Esta morfología es muy homogénea en los distintos géneros.

\section{DISCUSIÓN}

Los rasgos morfológicos de los dientes de los mesoterinos son bastante homogéneos entre las distintas especies descritas. De hecho, el material estudiado no permite en muchos casos diferenciar claramente si se trata de Typotheriopsis o Pseudotypotherium. Como se deduce de las comparaciones expuestas más arriba, los restos se asemejan tanto a uno como a otro género. Además, las especies del Huayqueriense argentino se conocen de forma incompleta. T. vanhouttei y $T$. jachalensis se conocen sólo por fragmentos maxilares. $T$. silveyrai se definió a partir de un cráneo bastante completo; también existe un fragmento mandibular, huesos postcraneales y algunos otros restos muy mal conservados. $P$. subinsigne cuenta con el cráneo tipo y un fragmento mandibular juvenil (" $P$. carlesi"). En el Museo "J. C. Moyano" de Mendoza, existen un cráneo y una mandíbula (MCNAM-PV 42) etiquetados como $P$. subinsigne que, aunque no consta la procedencia, es posible que procedan de las Huayquerías de San Carlos, igual que el holotipo de esta especie. De $P$. carhuense se conoce un fragmento maxilar juvenil y varios fragmentos mandibulares adultos.
1. Rostro: alargado y bajo (0); largo y más alto (1); corto, rodentiforme (2)

2. Estrechamiento de los premaxilares: ausente (0); poco acusado (1); muy acusado (2)

3. Escotadura anterior rostral (premaxilares): concavidad muy suave (0); en ángulo abierto (1); en ángulo cerrado (2); alta, amplia, en U (3)

4. Diastemas (superiores e inferiores): ausentes o poco desarrollados $(0)$; divergentes postero-lateralmente (1); paralelos o ligeramente convergentes (2); muy convergentes, convexos (3)

5. Apófisis postorbitarias: cortas (0), largas, transversales (1); largas, dirigidas hacia atrás (2)

6. Foseta suborbitaria y foramen infraorbitario: foseta ausente y foramen muy pequeño $(0)$; foseta insinuada y foramen pequeño (1); foseta desarrollada y foramen grande (2)

7. Arranque del arco cigomático: desde el nivel del M1 o M2 hacia atrás (0); perpendicular a M1 (1); perpendicular a M1 y con borde bicóncavo (2)

8. Placa cigomática: ausente o apenas desarrollada (0); poco desarrollada (1); muy desarrollada (2)

9. Escotadura postpalatina: estrecha, profunda y alejada del nivel de M3 (0); amplia y alejada de M3 (1); profunda hasta el nivel de M3 (2)

10. Escotadura occipital: ausente (0); presente (1)

11. Apófisis paraoccipitales: cortas (0); largas (1)

12. Dentición: mesodonta (0); protohipsodonta (1); euhipsodonta (2)

13. Imbricación dentaria: escasa (0); moderada (1); acusada (2)

14. I1: con esmalte continuo (0); con esmalte labial (1)

15. I1: implantación oblicua no procumbente $(0)$; implantación oblicua, procumbente (1); implantación más transversal, procumbente (2)

16. I1 ovalado (0); subtriangular, apuntado distalmente, con surco mesial (1); ancho, arriñonado, con surco lingual (2); ancho y aplastado labio-lingualmente, con dos surcos linguales (3)

17. I2-I3-C-P1/i2-i3-c-p1: desarrollados (0); reducidos, a veces ausentes en el adulto (1); siempre ausentes (2)

18. P2: desarrollado (0); reducido (1); ausente (2)

19. P4: subtriangular, corto, con foseta media (0); subtriangular, corto, sin foseta media (1); bilobulado, con pliegue corto y poco marcado (2); bilobulado, con pliegue bien desarrollado y patente (3)

20. M1-M2: subtriangulares, con foseta (0); con lóbulos anterior y posterior convergentes, encerrando el lóbulo medio que desaparece con el desgaste (1); no convergentes, con lóbulo medio largo (2)

21. M3: subtriangular, con foseta (0); trilobulado, con lóbulo medio poco diferenciado (1); con lóbulo medio corto, muy encerrado por los otros dos lóbulos (2); con lóbulo medio menos encerrado pero aún más corto que los otros (3)

22. Relación i1/i2: 1/1 (0); 1,5/1 (1); >=2/1 (2); 5/1 (3)

23. Sección del il: subcilíndrica (0); arriñonada, con suave surco lingual (1); subtriangular, con surco suave (2); trapezoidal con surco lingual amplio marcado (3); elíptica, con surco suave (4)

24. p3: desarrollado (0); ausente (1)

25. Molariformes inferiores: con fosétidas (0); sin fosétidas (1)

Tabla 4. Lista de caracteres considerados en el análisis cladístico de la subfamilia Mesotheriinae. Los caracteres $1,3,9,16$ y 23 se consideraron desordenados.

List of characters considered in the cladistic analysis of the subfamily Mesotheriinae. Characters 1, 3, 9, 16, and 23 were considered non-additive. 
Los datos disponibles ponen de manifiesto la gran similitud existente entre las tres especies huayquerienses de Typotheriopsis, $T$. silveyrai, $T$. vanhouttei y $T$. jachalensis, lo que nos hace suponer que se trata de una sola especie que está presente tanto en Mendoza como en La Pampa y San Juan, así como en San Luis con "Acrotypotherium". Por otra parte, tampoco nos parece justificada la separación del material huayqueriense de Pseudotypotherium en dos especies, proponiendo la sinonimia de $P$. carhuense y $P$. subinsigne. La prioridad de nombre corresponde, respectivamente, a $T$. silveyrai y a $P$. subinsigne.

El rasgo más claro para diferenciar las dos especies entre el material huayqueriense del centro de Argentina es la presencia o ausencia de surco lingual en el P4 y, según este carácter, tanto Typotheriopsis silveyrai como Pseudotypotherium subinsigne están presentes.

Para intentar dilucidar las relaciones filogenéticas existentes entre las distintas especies consideradas y comprobar si realmente constituyen grupos monofiléticos, hemos realizado un análisis cladístico de la subfamilia Mesotheriinae.

\section{ANÁLISIS CLADÍSTICO}

Para realizar el análisis cladístico de la subfamilia Mesotheriinae, hemos confeccionado una matriz de 25 caracteres (craneales y dentarios) y 13 taxones (Tablas 45), incluyendo como grupos externos la familia Archaeohyracidae y la subfamilia Trachytheriinae (Mesotheriidae), por ser los grupos considerados más afines a los mesoterinos. Los arqueohirácidos forman el grupo hermano de los mesotéridos (Reguero, 1999), mientras que los traquiterinos constituyen junto con los mesoterinos las dos únicas subfamilias de Mesotheriidae y ambas son consideradas como grupos hermanos. Dentro del grupo de estudio, se han excluido del análisis algunas de las especies descritas debido a la escasa información que proveen. Por ejemplo, las especies huayquerienses Typotheriopsis vanhouttei (Cattoi, 1943) y T. jachalensis (Rusconi, 1947) se basan en fragmentos maxilares que sólo permiten codificar cuatro de los 25 caracteres considerados y, como ya hemos comentado, las consideramos sinónimas de $T$. silveyrai. En Pseudotypotherium subinsigne se han combinado los caracteres de $P$. carhuense y $P$. subinsigne, ya que también establecemos su sinonimia. En el caso de las especies descritas en el yacimiento bonaerense de Monte Hermoso, las diferencias establecidas entre ellas son sutiles y hemos preferido considerar todo el conjunto de este yacimiento (de edad Montehermosense) como un único taxón, combinando los caracteres de los distintos restos descritos (Ameghino, 1887, 1904; Burmeister, 1888; Rovereto, 1914; Kraglievich, 1934), ya que además ninguna de ellas ha sido ampliamente caracterizada. Este mismo criterio podría aplicarse a las especies asignadas al Pleistoceno, pero en estos casos las descripciones son más completas y hemos preferido incluir los taxones separadamente y analizar el resultado obtenido. Entre

$\begin{array}{lrrrrr} & \text { Carácter } & 1 & 11111 & 11112 & 22222 \\ \text { Taxón } & 12345 & 67890 & 12345 & 67890 & 12345\end{array}$

$\begin{array}{lllllll}\text { Archaeohyracidae } & 00000 & 000 \mathrm{~A} 0 & 0 \mathrm{~A} 100 & 00000 & 00000 \\ \text { Trachytherinae } & 10101 & 20111 & 01111 & 111 \mathrm{~A} 1 & 1010 \mathrm{~A} \\ \text { E. lehmannistchei } & 21 ? 1 ? & 10211 & ? 2001 & 22222 & 21211 \\ \text { E. superans } & 2121 ? & 10211 & ? 2001 & 22212 & 3 ? ? ? ? \\ \text { T. chasicoensis } & 21 ? 22 & 2 \mathrm{~A} 201 & 12001 & 22212 & 22211 \\ \text { T. silveyrai } & 21222 & 21201 & 120 ? 1 & ? 2212 & 2 ? ? ? ? \\ \text { P. subinsigne } & 21 ? 21 & 212 \mathrm{~A} 1 & 12201 & ? 22 \mathrm{C} 2 & \mathrm{C} 2311 \\ \text { P. exiguum } & 2 ? ? ? 1 & ? 121 ? & ? 22 ? ? & ? ? ? 32 & 32 ? 11 \\ \text { M. hystatum } & 21231 & ? 0201 & 122 ? 1 & ? 2232 & 3 ? ? 11 \\ \text { M. cristatum } & 22332 & 02221 & 12202 & 22232 & 33311 \\ \text { M. pachignathum } & 21 ? 22 & 01231 & 12202 & 22232 & 33 ? 11 \\ \text { M. maendrum } & 22232 & 01221 & 12202 & 32232 & 33411 \\ \text { Plesiotypotherium } & 21122 & 21211 & 12001 & 222 \mathrm{~B} 2 & 31211\end{array}$

Tabla 5. Matriz de datos utilizada para el análisis cladístico de la subfamilia Mesotheriinae, incluyendo dos grupos externos (Archaeohyracidae y Trachytheriinae), 11 taxones de Mesotheriinae y 25 caracteres. A, estados de carácter 0 y 1 ; B, estados 1 y 2 ; C, estados 2 y 3 .

Data matrix used in the cladistic analysis of the subfamily Mesotheriinae, including two out-groups (Archaeohyracidae and Trachytheriinae), 11 Mesotheriinae taxa, and 25 characters. A, character states 0 and $1 ; B$, states 1 and 2; C, states 2 and 3.

ellas, Mesotherium maendrum (Ameghino, 1887) fue propuesta como sinónima de M. cristatum (Serrés, 1874) por Mercerat (1891), lo cual no fue aceptado por Rovereto (1914). En el caso del género boliviano Plesiotypotherium, Villarroel (1974) describió dos especies, $P$. achirense y $P$. majus, cuya diferencia reside en la mayor talla y robustez de la segunda, por lo que en el análisis las hemos considerado a nivel genérico.

El análisis se ha llevado a cabo a través del programa Pee-Wee 2.0 (Goloboff, 1993), que permite incluir en la matriz de datos los distintos estados de un mismo carácter en caso de especies con variación morfológica para un carácter dado, evitando así la inclusión de datos faltantes (?) que restan información. Este programa busca los árboles filogenéticos más simples utilizando la opción de pesado de caracteres.

Los caracteres $1,3,9,16$ y 23 se consideraron como desordenados o no aditivos, al no presentar una secuencia lógica a priori entre los estados de carácter definidos. El programa, por su parte, trata como no aditivos todos aquellos caracteres que presentan multiestados en alguno de los taxones analizados.

El análisis de la matriz de datos proporcionó un único cladograma $($ fit $=177,5)$. El nodo basal del grupo de estudio está conformado por una tricotomía que incluye las dos especies de Eutypotherium y el resto de los mesoterinos reunidos en un clado. Esta topología parece indicar que dos de las resoluciones de la tricotomía podrían establecer que el género Eutypotherium constituye 
un grupo parafilético. Sin embargo, existen varios estados de carácter que apoyarían que el género Eutypotherium sí constituye un grupo monofilético y que es el grupo hermano de los restantes mesoterinos. La explicación de por qué este género no es dado como monofilético por el programa Pee Wee es que bajo la opción "colapso estricto" (collapse strictly) agrupa en un mismo nodo aquellas ramas que podrían estar soportadas por caracteres ambiguos. Sin embargo, si se acepta la opción "colapso parcial" (collapse slightly) del programa, sí reconoce al género Eutypotherium como grupo monofilético soportado por una sinapomorfía. En consecuencia, elegimos este cladograma como el que mejor refleja la filogenia de la subfamilia Mesotheriinae (Fig. 7).

La monofilia de la subfamilia Mesotheriinae queda apoyada por 14 sinapomorfías (Fig. 7, nodo 2): estrechamiento de los premaxilares poco acusado; diastemas divergentes postero-lateralmente; placa cigomática muy desarrollada; dentición euhipsodonta; imbricación dentaria escasa; I1 ancho, arriñonado, con surco lingual; I2-I3-C-P1/i2-i3-c-p1 ausentes; P2 ausente; P4 subtriangular, corto, sin foseta; M1-M2 con lóbulo medio largo; relación i1/i2 de 1,5/1; i1 subtriangular, con surco suave; p3 ausente; y molariformes sin fosétidas. Entre estas sinapomorfías, hay algunas que con la opción de "colapso estricto" resultarían ambiguas, como es el caso del carácter 25 (fosétidas en los molariformes inferiores), ya que si bien todos los mesoterinos carecen de fosétidas $\left(25^{1}\right)$, el carácter es variable en los Trachytheriinae.

En la topología elegida, las dos especies de Eutypotherium comparten la foseta suborbitaria insinuada y el foramen pequeño (6') (Fig. 7, nodo 3).

El resto de los mesoterinos comparte cuatro sinapomorfías (Fig. 7, nodo 4), los diastemas paralelos o ligeramente convergentes, las apófisis postorbitarias largas y hacia atrás y el arranque del arco cigomático perpendicular a M1. Dentro de este gran grupo, el primer taxón que se separa es Plesiotypotherium, constituyendo el grupo hermano de los demás mesoterinos que comparten, a su vez, dos sinapomorfías (Fig. 7, nodo 5), la escotadura anterior rostral en ángulo cerrado y la relación entre los incisivos inferiores igual o mayor a 2/1. Estos taxones forman a su vez dos clados principales.

Uno está compuesto por las especies de Typotheriopsis, que comparten tres sinapomorfías, la foseta suborbitaria desarrollada y el foramen grande, la escotadura postpalatina estrecha, profunda y alejada del M3 y el M3 con lóbulo medio corto, muy encerrado por los otros dos (Fig. 7, nodo 6). Typotheriopsis aparece así como grupo hermano del clado formado por los géneros Pseudotypotherium y Mesotherium que comparten tres sinapomorfías (Fig. 7, nodo 7), imbricación dentaria acusada, P4 bilobulado, con pliegue bien desarrollado y sección del i1 elíptica, con surco suave. Estos dos últimos géneros constituyen unidades monofiléticas. Las especies de Pseudotypotherium comparten una sinapomorfía, apófisis postorbitarias largas y transversales $\left(5^{1}\right)$, pero las relaciones filogenéticas entre ellas no quedan definidas y las tres especies forman una tricotomía.

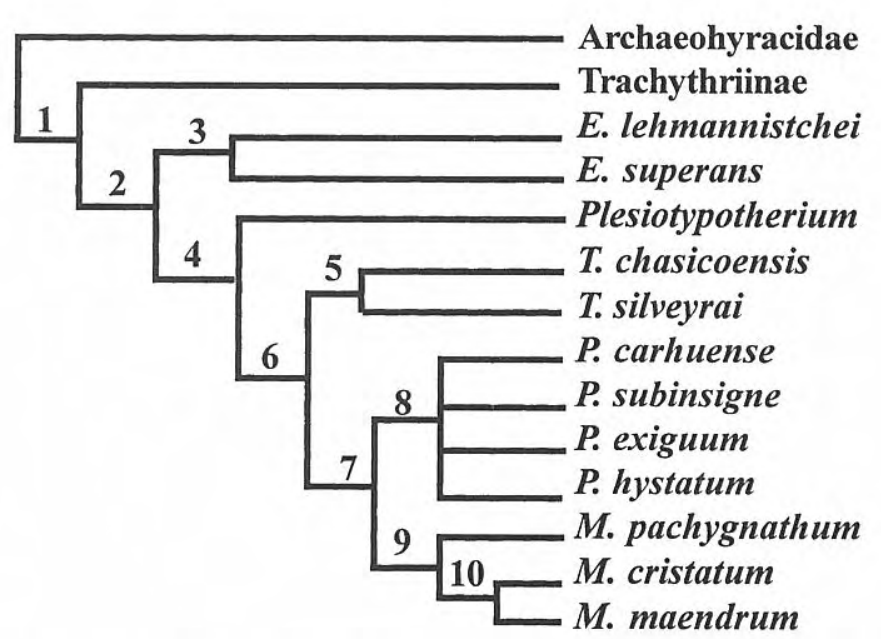

Figura 7. Cladograma obtenido en el análisis cladístico de la subfamilia Mesotheriinae con la opción “colapso parcial". Nodo 1: 5', 8', 9', 10', 12', 15', $17^{1}, 18^{1}, 20^{1}$; Nodo $2: 2^{1}, 4^{\prime}, 8^{2}, 12^{2}, 13^{0}, 16^{2}, 17^{2}$, $18^{2}, 19^{1}, 20^{2}, 22^{1}, 23^{1}, 24^{1}, 25^{\prime}$; Nodo 3: 6'; Nodo 4: $4^{2}, 5^{2}, 7^{1}$; Nodo 5: $3^{2}, 22^{2}$; Nodo 6: $6^{2}, 9^{0}, 21^{2}$; Nodo 7: $13^{2}, 19^{3}, 23^{3}$; Nodo 8: 5'; Nodo 9: $6^{0}, 15^{2}$, $22^{3}$; Nodo 10: $2^{2}, 4^{3}, 9^{2}$.

Obtained cladogram in the cladistic analysis of the subfamily Mesotheriinae with the option "collapse slightly".

Las especies del género Mesotherium comparten tres sinapomorfías (Fig. 7, nodo 9): foseta suborbitaria ausente y foramen muy pequeño, lo cual constituye una reversión al carácter plesiomorfo; el I1 con implantación bastante transversal y la relación i1/i2 de 5/1 $\left(22^{3}\right)$. Dentro de este grupo, $M$. pachygnathum constituye la especie basal y el clado formado por $M$. cristatum y $M$. maendrum está justificado por tres sinapomorfías (nodo 10): estrechamiento de los premaxilares muy acusado, los diastemas muy convergentes, convexos; y la escotadura postpalatina profunda hasta el nivel del M3.

Si reflejamos la filogenia obtenida en una clasificación, vemos que todos los géneros considerados están bien justificados como grupos monofiléticos, teniendo en cuenta la consideración hecha para Eutypotherium.

La relación entre las especies del género Pseudotypotherium no queda resuelta. La forma montehermosense $P$. exiguum es muy semejante a la especie huayqueriense $P$. subinsigne, pero en ella se observa el establecimiento general del P4 con surco lingual bien patente y el desarrollo del lóbulo medio de M3, caracteres (19 y 21) que son variables en $P$. subinsigne. $P$. hystatum es la especie más reciente del género, del Chapadmalalense de Miramar (Buenos Aires), y se distingue de las otras por un paralelismo con dos especies de Mesotherium (diastemas muy convergentes, convexos, $4^{3}$ ) y una reversión (arranque del arco cigomático desde M1 o M2 hacia atrás, $7^{\circ}$ ).

Mesotherium cristatum y $M$. maendrum están diferenciadas por dos autapomorfías cada una. La primera 
especie presenta la escotadura anterior rostral alta, en forma de $\mathrm{U}\left(3^{3}\right)$ y el arranque del arco cigomático con borde bicóncavo $\left(7^{2}\right)$, mientras que la segunda tiene el I1 aplastado labio-lingualmente, con dos surcos linguales $\left(16^{3}\right)$ y la sección del I1 elíptica, con surco suave $\left(23^{4}\right)$.

\section{CONCLUSIONES}

El estudio anátómico comparativo de los restos de mesotéridos del Huayqueriense de La Pampa, procedentes principalmente de la Formación Cerro Azul, ha permitido identificar las especies Typotheriopsis silveyrai y Pseudotypotherium subinsigne.

Al mismo tiempo, la revisión de distintos restos de mesotéridos pone de manifiesto la gran similitud existente entre las tres especies huayquerienses de Typotheriopsis, proponiendo la sinonimia de $T$. silveyrai, T. vanhouttei y T. jachalensis. Por otra parte, tampoco nos parece justificada la separación del material huayqueriense de Pseudotypotherium en dos especies y establecemos la sinonimia de $P$. carhuense y $P$. subinsigne. La prioridad de nombre corresponde, respectivamente, a $T$. silveyrai y a $P$. subinsigne.

El análisis cladístico de la subfamilia Mesotheriinae apoya la monofilia tanto de la propia subfamilia como de los distintos géneros analizados, siendo la relación filogenética de las especies de Pseudotypotherium la que queda irresuelta. Eutypotherium es el grupo hermano del resto de los mesoterinos, mientras que Typotheriopsis lo es de los géneros Pseudotypotherium y Mesotherium.

\section{AGRADECIMIENTOS}

Expresamos nuestro sincero agradecimiento a Marcelo Reguero, de la Facultad y Museo de La Plata, a José Bonaparte, del Museo de Ciencias Naturales "Bernardino Rivadavia" de Buenos Aires, a Graciela Esteban y Norma Nasif, de la Facultad de Ciencias e Instituto Miguel Lillo de Tucumán, y a Clara Abal, subdirectora del Museo "J. C. Moyano" de Mendoza, por facilitarnos la consulta de las colecciones a su cargo. A Sergio Roig, por la revisión del manuscrito y en especial por las sugerencias realizadas sobre el análisis cladístico. A G. Visconti, quien colaboró en las tareas de campo. Este trabajo fue financiado por la Facultad de Ciencias Exactas y Naturales de la Universidad Nacional de La Pampa.

\section{BIBLIOGRAFÍA}

Ameghino, F. 1887. Apuntes preliminares sobre algunos mamíferos extinguidos de Monte Hermoso. Obras Completas y Correspondencia Científica, 5 (1916) (44), 339-353.

Ameghino, F. 1891. Sobre algunos nuevos restos de mamíferos fósiles, recogidos por el señor Manuel B. Zavaleta en la formación miocena de Tucumán y
Catamarca. Revista Argentina de Historia Natural, 1 (2), 88-101.

Ameghino, F. 1904. Nuevas especies de mamíferos cretáceos y terciarios de la República Argentina. Anales de la Sociedad Científica Argentina, 56, 162-175.

Bond, M., Cerdeño, E. y López, G. 1995. Los ungulados nativos de América del Sur. In: Evolución biológica y climática de la región pampeana durante los últimos cinco millones de años. Un ensayo de correlación con el Mediterráneo Occidental. (Eds. T. Alberdi, G. Leone y E. Tonni). Museo Nacional de Ciencias Naturales y Consejo Superior de Investigaciones Científicas, Madrid, 259-275.

Burmeister, C. V. 1888. Relación de un viaje a la Gobernación de Chubut. Anales del Museo Nacional de Buenos Aires, 3, 175-222.

Cabrera, A. 1937. Notas sobre el suborden "Typotheria". Notas del Museo de La Plata, 2, Paleontología (8), $17-$ 43.

Cabrera, A. 1939. Los géneros de la Familia Typotheriidae. Physis, 14, 359-372.

Cabrera, A. y Kraglievich, L. 1931. Diagnosis previas de los ungulados fósiles del Arroyo Chasicó. Notas Preliminares del Museo de La Plata, 1, 107-113.

Cattoi, N. 1943. Otras observaciones sobre Typotheriidae. Physis, 19, 356-361.

Esteban, G. I. y Nasif, N. L. 1996. Nuevos Dasypodidae (Mammalia, Xenarthra) del Mioceno tardío del Valle del Cajón, Catamarca, Argentina. Ameghiniana, 33, 327 334.

Franchi, M., Nullo, F., Sepúlveda, E. y Uliana, M. 1984. Las sedimentitas terciarias. IX Congreso Geológico Argentino, Relatorio, 1(9), 215-266.

Francis, J. C. 1965. Los géneros de la subfamilia Mesotheriinae (Typotheria, Notoungulata) de la República Argentina. Boletín del Laboratorio de Paleontología de Vertebrados (Montevideo), 1 (1), 1-31.

Goin, F. y Montalvo, C. I. 1988. Revisión sistemática y reconocimiento de una nueva especie del género Thylatheridium Reig (Marsupialia, Didelphidae). Ameghiniana, 25, 161-167.

Goin, F. J., Montalvo, C. I. y Visconti, G. 1997. Los marsupiales de la Formación Cerro Azul (Mioceno tardío), Provincia de La Pampa, Argentina. Ameghiniana, 34, 536.

Goin, F. J., Montalvo, C. I. y Visconti, G. (en prensa). Los marsupiales (Mammalia) del Mioceno superior de la Formación Cerro Azul (Provincia de La Pampa, Argentina). Estudios Geológicos.

Goloboff, P. 1993. PIWE: parsimony and implied weights. MS-Dos program and documentation. Programa de computación distribuido por el autor.

Kraglievich, L. 1934. La antigüedad pliocena de las faunas de Monte Hermoso y Chapadmalal, deducidas de su comparación con las que le precedieron y sucedieron. Montevideo, Imprenta El Siglo Ilustrado. Obras Completas, III, 293-433.

Linares, E., Llambías, E. y Latorre, E. 1980. Geología de la provincia de La Pampa, República Argentina y geocronología de sus rocas metamórficas y eruptivas. 
Revista de la Asociación Geológica Argentina, 35, 87146.

Marshall, L. and Patterson, B. 1981. Geology and Geochronology of the mammal-bearing Tertiary of the Valle de Santa María and río Corral Quemado, Catamarca Province, Argentina. Fieldiana (Geology), 9, $1-80$.

Mercerat, A. 1891. Apuntes sobre el género Typotherium. Revista del Museo de La Plata, 2, 74-80.

Montalvo, C. I. y Casadío, S. 1988. Presencia del género Palaeoctodon (Rodentia, Octodontidae) en el Huayqueriense (Mioceno tardío) de la Provincia de La Pampa. Ameghiniana, 25, 111-114.

Montalvo, C. I., Visconti, G., Pugener, L. y Cardonatto, M. 1995. Mamíferos de edad Huayqueriense (Mioceno tardío), Laguna Chillhué, Provincia de La Pampa. IV Jornadas de Geología y Geofísica Bonaerenses, Actas, 1, 73-79.

Montalvo, C. I., Cardonatto, M., Visconti, G., Verzi, D. H. y Vucetich, M. G. 1996. Vertebrados de la Formación Cerro Azul (Mioceno tardío) del valle de Quehué, Provincia de La Pampa, Argentina. VI Jornadas Pampeanas de Ciencias Naturales, Actas, 159-165.

Moreno, F. P. y Mercerat, A. 1891. Paleontología. Revista del Museo de La Plata, 1, 222-236.

Ortega Hinojosa, E. 1967. Descripción de los restos de un Scelidotheriinae (Edentata, Mylodontidae) de edad Huayqueriense. Algunas consideraciones en torno a la filogenia de los Scelidotheriinae. Ameghiniana, 5, 109120.

Pascual, R. y Bocchino, A. 1963. Un nuevo Borhyaeninae (Marsupialia) del Plioceno medio de Hidalgo (La Pampa). Ameghiniana, 3, 97-107.

Pascual, R. y Bondesio, P. 1982. Un roedor Cardiatheriinae (Hydrochoeridae) de la Edad Huayqueriense (Mioceno tardío) de La Pampa. Sumario de los ambientes terrestres en la Argentina durante el Mioceno. Ameghiniana, 19, 19-35.

Pascual, R., Ortega, E. J., Gondar, D. y Tonni, E. P. 1965. Las edades del Cenozoico mamalífero de la Argentina, con especial atención a aquéllos del territorio bonaerense. Anales de la Comisión de Investigación Científica de Buenos Aires, 6, 165-193.

Pascual, R., Ortega-Hinojosa, E. J., Gondar, D. y Tonni, E. P. (Eds.) 1966. Paleontografía bonaerense. IV. Vertebrata. A.V. Borrello, La Plata. 202 pp., 101 láms.

Reguero, M. A. 1999. El problema de las relaciones sistemáticas y filogenéticas de los Typotheria y Hegetotheria (Mammalia, Notoungulata): análisis de los taxones de Patagonia de la edad-mamífero Deseadense (Oligoceno). Tesis Doctoral Inédita. Universidad Nacional de Buenos Aires, Fac. de Ciencias Exactas y Naturales. 350 pp.
Rovereto, C. 1914. Los estratos araucanos y sus fósiles. Anales del Museo Nacional de Historia Natural de Buenos Aires, 25, 1-250.

Rusconi, C. 1934. Algunos fósiles terciarios de la Gobernación de La Pampa Argentina. Revista Chilena de Historia Natural, 38, 104-107.

Rusconi, C. 1936. Restos de mamíferos terciarios de San Luis. Boletín Paleontológico de Buenos Aires, 6, 1-4.

Rusconi, C. 1947. Nueva especie de tipotérido de Jáchal (San Juan). Anales de la Sociedad Científica Argentina, 143, 247-250.

Serrés, M. 1874. De l'ostéographie du Mesotherium et ses affinités zoologiques. Comptes Rendus Séances de l'Académie de Sciences, 65 (4), 140-148.

Uliana, M. 1979.Geología de la región comprendida entre los ríos Colorado y Negro, provincias de Neuquen y Río Negro. Universidad Nacional de La Plata, Tesis Doctoral Inédita $\mathrm{n}^{\circ} 371,117 \mathrm{pp}$.

Verzi, D. H., Montalvo, C. I. y Vucetich, M. G. 1991. Nuevos restos de Xenodontomys simpsoni Kraglievich y la sistemática de los más antiguos Ctenomyinae (Rodentia, Octodontidae). Ameghiniana, 28, 325-331.

Verzi, D. H., Vucetich, M. G. and Montalvo, C. I. 1994. Octodontid-like Echimyidae (Rodentia): an upper Miocene episode in the radiation of the family. Palaeovertebrata, 23, 199-210.

Verzi, D. H., Vucetich, M. G. y Montalvo, C. I. 1995. Un nuevo Eumysopinae (Rodentia, Echimyidae) del Mioceno tardío de la provincia de La Pampa y consideraciones sobre la historia de la subfamilia. Ameghiniana, 32, 191-195.

Verzi, D. H., Montalvo, C. I. y Vucetich, M. G. 1999. Afinidades y significado evolutivo de Neophanomys biplicatus (Rodentia, Octodontidae) del Mioceno tardíoPlioceno temprano de Argentina. Ameghiniana, 36, 8390.

Villarroel, C. 1974. Les mésothérinés (Notoungulata, Mammalia) du Pliocène de Bolivie. Leurs rapports avec ceux d'Argentine. Annales de Paléontologie, 60, $245-$ 285.

Visconti, G., Montalvo, C. I., Cardonatto, M. C. y Pugener, L. A. 1996. Análisis sedimentológico e interpretación paleoambiental de la Formación Cerro Azul (Mioceno tardío) en el Valle Argentino, Provincia de La Pampa. Comunicaciones VI Jornadas Pampeanas de Ciencias Naturales, Santa Rosa, 86-88.

Zetti, J. 1967. Sobre la presencia del género Marmosa (Didelphidae, Marsupialia) en sedimentos de la Formación Epecuén (Plioceno medio). Ameghiniana, 5, $169-173$.

Zetti, J. 1972. Los mamíferos fósiles de edad huayqueriense (Plioceno medio) de la región pampeana. Tesis Doctoral Inédita. Universidad Nacional de La Plata, 122 pp. 